\title{
Interphase interaction of near-wall liquid film with co-current gas flow inside a nozzle
}

\author{
Yuri Vyazov, Victor Prihodko, Igor Yarygin and Vyacheslav Yarygin ${ }^{a}$ \\ Kutateladze Institute of Thermophysics SB RAS, Rarefied Gases Laboratory, 630090, Lavrentieva ave. 1, \\ Novosibirsk, Russia
}

\begin{abstract}
The results of experimental study on high-velocity co-current gas flow interaction with near-wall liquid film are presented in the paper. Local parameters of near-wall liquid film are measured with the help of capacity-type probes. It is shown that co-current gas flow has strong influence on near-wall liquid film, leading to intensive wave formation, detachment of droplets from the film surface and their entrainment by the gas flow. A model for the film motion with co-current gas flow, linking together thickness and velocity of the film with value of shearing stress at gas-liquid boundary, is suggested.
\end{abstract}

\section{Introduction}

Flow of liquid in the form of thin films (with thickness less than $1 \mathrm{~mm}$ ) is widely used in various heat-andmass exchangers that involve condensation of stationary and moving vapor, absorption or desorption of gas, drying, distillation, fractioning, chemical processes, etc. Another important application of film flows is protection of walls from the high-temperature gas flow, for example, protection of liquid-propellant thrusters combustion chamber and supersonic nozzle using the near-wall fuel film.

Thin film flows attracted a great deal of attention recently. The most thorough and extended studies are concerned with gravitation films. Important role in such flows is played by the mechanisms of instability leading to evolution and interaction of waves of various character. Thermocapillary instability effect and phase transformation can also be significant. In such phenomena condensation has a stabilizing effect, and evaporation destabilizes the flow. The results of these studies can be found for example in monographs $[1,2]$.

The stress film flows are studied extensively in experiments. However, these experiments are carried out at relatively low velocities of the co-current flow of about $10 \mathrm{~m} / \mathrm{s}$. Interaction of the nearwall film with the co-current gas flow is mainly a function of the flow parameters, such as velocity, density, and static pressure. Even at low flow velocities, liquid film loses its stability, and capillary waves are formed on its surface, whereas, without the co-current flow, the film flow is waveless. An increase in velocity of the co-current gas flow facilitates interfacial interaction and leads to formation of 2D and 3D waves on the film surface. Beginning with some values of gas flow velocity and density (Weber number), droplets get detached from the film surface and entrained by the co-current flow [3].

Another special feature of such two-phase flows is interaction of the near-wall film with the co-current gradient flow, for example inside nozzles. A significant drop in static pressure in the

${ }^{a}$ Corresponding author : yarygin@itp.nsc.ru 
flow (approximately, by the factor of 2 for Mach number 1, and by the factor of 30 for Mach number 3) can cause the situation, when the static pressure in the flow above the film becomes lower than the liquid saturated vapor pressure. This leads to film boiling-up and evaporation, as well as formation of a transverse vapor flow from the film surface to the boundary layer. Similar to injection through a permeable wall into the boundary layer, this vapor flow can essentially affect the value of interface friction causing a decrease in the shearing stress on the gas-liquid interface in comparison with the modes of gas-film interaction without evaporation. Currently there are no complete studies with accurate and reliable data on the values of shearing stress on the gas-liquid interface under the condition of high relative velocities of gas flow. Existing experimental studies are focused on the measurement of flow integral characteristics and averaged parameters of waves without taking their non-stationary character into consideration $[4,5]$.

Our early studies were devoted to solving the problem of the International Space Station (ISS) external contamination by the jets from orientation thrusters, in which the fuel film is used for nozzle walls cooling. Experimental studies on modeling operation of orientation thrusters showed that behind the nozzle exit complicated flow structure of droplet phase takes place, and droplets are dispersed at angles up to $180^{\circ}$ relative to jet axis. Such flows of droplets are the reason of the ISS external surface contamination [6]. This paper presents the results of experimental studies on the interaction of cocurrent gas flow with near-wall liquid film for supersonic nozzle in a wider range as compared to the earlier modeling experiments of the flow parameters on the magnitude of gas flow pressure above near-wall liquid film. Dodecane, as well as ethanol were used as working liquids, which allowed us to carry experiments for liquids with significantly different values of saturated vapor pressure.

\section{Experimental setup and measurement techniques}

Measurements of local parameters of a near-wall liquid film (film thickness and film velocity) in the present study were carried out with the help of capacity-type probes [7]. Film thickness and velocity were measured near the exit edge of the supersonic nozzle (geometrical Mach number $\mathrm{M}=3$ ) with 10 $\mathrm{mm}$ throat diameter, $20 \mathrm{~mm}$ exit diameter and $20 \mathrm{~mm}$ nozzle length (Fig. 1). Four probes were mounted around the nozzle for film thickness measurements (results were avaraged by four probes), and two consequently mounted probes were used for film velocity measurements.

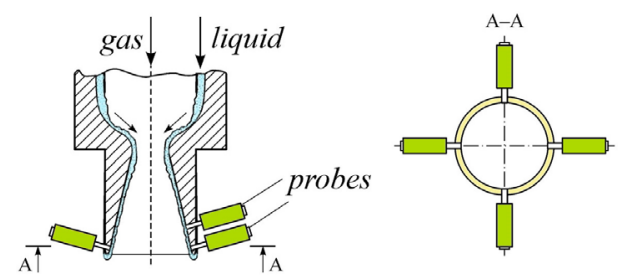

Figure 1. Scheme of test section.

Purified air was used as a working gas, and ethanol and dodecane were used as working liquids. Initially both gas (flow rate from 2 to $20 \mathrm{~g} / \mathrm{s}$ ) and liquid (flow rate from 0.5 to $2 \mathrm{~g} / \mathrm{s}$ ) were at the room temperature. Gas flow regime was turbulent. All experiments were carried out at the large-scale vacuum gas-dynamical setup VIKING of Kutateladze Institute of Thermophysics SB RAS (volume of vacuum chamber is about $150 \mathrm{~m}^{3}$ ). Ethanol and dodecane were used in experiments because their main physical properties (density, specific evaporation heat, viscosity, surface tension) are similar to properties of the rocket fuel. At the same time they differ significantly in saturated vapor pressure. Ethanol has rather high vapor pressure equal to $5.86 \mathrm{kPa}$ at $20^{\circ} \mathrm{C}$ and evaporates easily in vacuum. Dodecane has much lower saturated vapor pressure $0.0097 \mathrm{kPa}$ at $20^{\circ} \mathrm{C}$ and almost does not evaporate.

A great deal of attention in our research was paid to calibration of the capacity-type probes. We developed the method with use of dielectric plugs, which were tightly inserted into the channel and had a groove of certain depth over the probe, filled with liquid. The method allowed us to obtain 
calibration curves for probes taking into account their placement on the channel surface, and to have liquid film thicknesses measurements margin of error less than $10 \%$.

\section{Results}

Ethanol film thickness diagram, measured by capacity-type probes is shown in Fig. 2. Solid line corresponds to the case when the film was not boiling. Stagnation pressure of gas in the nozzle was equal $p_{0}=100 \mathrm{kPa}$, and pressure of gas over the liquid film near the nozzle exit was about $3.3 \mathrm{kPa}$. Dashed line corresponds to the case when the film was boiling. Stagnation pressure in these experiments was equal to $p_{0}=13 \mathrm{kPa}$ and pressure over the liquid film was $0.43 \mathrm{kPa}$, respectively. Reynolds numbers in both cases were $\operatorname{Re}_{l}=14$. One can see that evaporation and boiling have strong influence on the behavior of the film, causing large fluctuations and increase of its mean thickness.

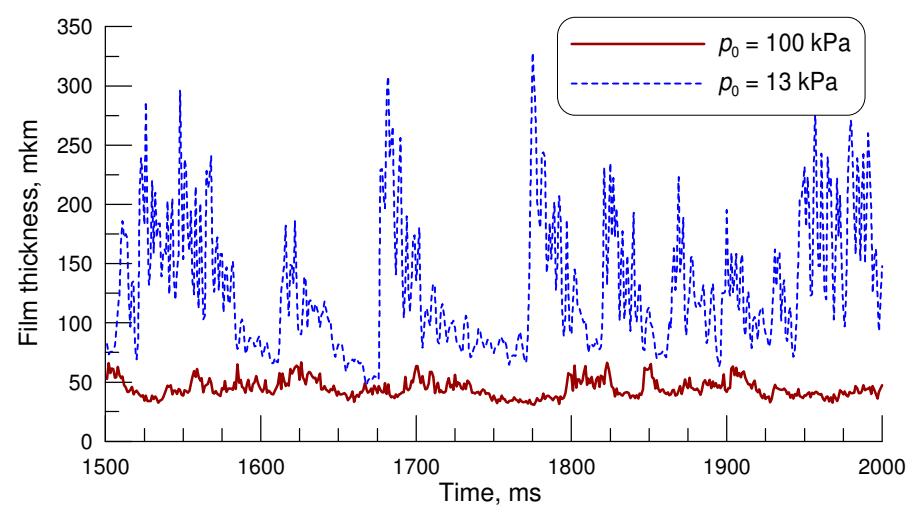

Figure 2. Ethanol film thickness without boiling $\left(p_{0}=100 \mathrm{kPa}\right)$ and with boiling $\left(p_{0}=13 \mathrm{kPa}\right)$.

Experiments allowed us to obtain mean film thickness and velocity inside the channel. It is shown that film velocity both for ethanol and dodecane increases from $0.2 \mathrm{~m} / \mathrm{s}$ for $\operatorname{Re}_{\text {gas }}=3.5 \cdot 10^{4}$ up to 0.8 $\mathrm{m} / \mathrm{s}$ for $\operatorname{Re}_{\text {gas }}=3.3 \cdot 10^{5}$, where Reynolds number $\operatorname{Re}_{\text {gas }}$ is calculated by the critical diameter of the nozzle. Measured data for ethanol and dodecane film thickness are shown in Fig. 3.
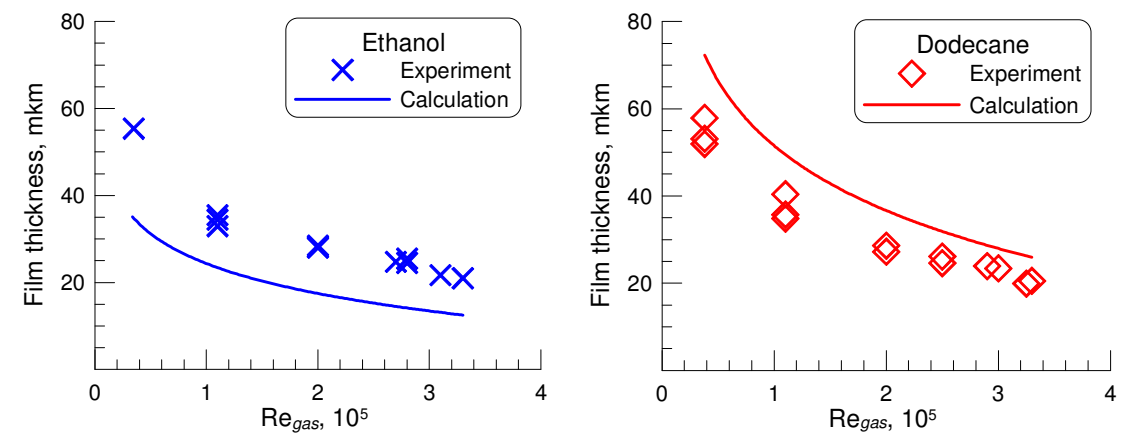

Figure 3. Dependence of liquid film thickness on Reynolds number of gas $\mathrm{Re}_{\text {gas }}$ for ethanol and dodecane.

The solid lines in these figures indicate mean film thicknesses $\delta$ calculated for the experimental conditions with the help of the model [8]:

$$
\delta=\sqrt{\frac{m \mu}{\pi R \rho \tau}},
$$

where $m, \mu, \rho$ - liquid flow rate, viscosity and density, respectively, $R$ - channel radius, $\tau$ - shearing stress at interface border. For these calculations it is necessary to know the value of shearing stress $\tau$. 
In our model the value of shearing stress $\tau$ at interface border is assumed as shearing stress $\tau_{w}$ on the nozzle wall without the film. Corresponding dependencies for turbulent boundary layer with pressure gradient in the external flow and absence of laminar region were taken from the reference-book, and they were as follows:

$$
C_{f}=\frac{2 \tau}{\rho V^{2}}=0,0131 \mathrm{Re}^{* *-1 / 6}, \text { where } \operatorname{Re}^{* *} \text { is determined as } \operatorname{Re}^{* * 7 / 6}=\frac{0,0076}{v V^{2,75}} \int_{0}^{x} V^{3,75} d x
$$

Our model despite its simplicity allowed us to estimate the film thickness and velocity under the motion inside the nozzle with the co-current gas flow. At the same time one can see that calculation results for dodecane are above experimental data and calculation results for ethanol are below experimental data. We believe that this can be explained by the influence of evaporation and wave formation that reduce and increase shearing stress respectively. For dodecane under considered conditions the main interaction processes between the co-current flow and the film are wave formation and droplet detachment, and the effect of film boiling is insignificant. Both wave formation and droplet detachment should increase $\tau$ at the interface in comparison with $\tau$ set in calculations on a smooth solid wall. For ethanol under considered conditions boiling plays a significant role. It follows from comparison of calculation and experimental data, shown in Fig. 3, that at film boiling $\tau$ should decrease, i.e., change in the opposite direction in comparison with a change in $\tau$ at wave formation and droplet detachment. These arguments are plausible, if we accept the existence of the analogy between the processes of evaporation and substance injection through a permeable wall.

\section{Conclusion}

Experimental study on interaction of high-velocity co-current gas flow with near-wall liquid film in supersonic nozzle was carried out. Local parameters of near-wall films - wave characteristics, thickness, velocity with high time resolution of $1 \mathrm{kHz}$ (gas flow velocity up to $540 \mathrm{~m} / \mathrm{s}$ ) were measured with the help of capacity-type probes. We have demonstrated that character of interaction (wave dispersion, evaporation, detachment of droplets from the film surface) is mainly determined by gas flow parameters (primarily, velocity and density) and physical properties of liquid (viscosity and saturated vapor pressure). The model of gas flow interaction with near-wall film was suggested. This model allows estimation of the shearing stress increase at the interface border under conditions of wave formation compared to thin laminar film with the help of measured values of thickness and velocity.

\section{Acknowledgments}

The study was supported by the Russian Foundation for Basic Research, research project No. 16-0800436.

\section{References}

1. S.V. Alekseenko, V.E. Nakoryakov V.E., and B.G. Pokusaev, Wave Flow of Liquid Films (1994).

2. S. Kalliadasis, C. Ruyer-Quil, B. Scheid, and M. G. Velarde, Falling Liquid Films, (2012).

3. I.I. Gogonin, J. Eng. Phys. Thermoph., 77, 454-470 (2004).

4. H. Han, Z. Zhu, K. Gabriel, Nucl. Engin. Des., 236, 2580-2588 (2006).

5. D.A. McNeil, A.D. Stuart, Int. J. of Heat and Fluid Flow, 25, 58-73 (2004).

6. V. Yarygin, Yu. Gerasimov, A. Krylov, V. Prikhodko, and I. Yarygin, Microgravity Sci. Technol., 23, 15-23 (2011).

7. V. Prikhodko, I. Yarygin and V. Yarygin, Microgravity Sci. Technol., 21, 277-281 (2009).

8. I.V. Yarygin, V.F. Levchenko, Proc. ICMAR, 4, 314-318 (2004). 